\title{
Smart Navigational Shoes for Bikers/Cyclists
}

\author{
Ganesh Deshmukh \\ Professor \\ PCCOE, Akurdi \\ Pune, Maharashtra, India
}

\author{
Nikhil Londhe \\ PCCOE, Akurdi \\ Pune, Maharashtra, India
}

\author{
Vedant Gawade \\ PCCOE, Akurdi \\ Pune, Maharashtra, India
}

\author{
Vedant Godse \\ PCCOE, Akurdi \\ Pune, Maharashtra, India
}

\begin{abstract}
The present solutions for the navigational purposes that are available in the market are not tailored specifically for the needs of bikers/cyclists. Hence, in this system we design a system based in a shoe that guides the motorcyclist along the route from source to destination. Since the system is implemented in shoes we use a battery for power supply. Bluetooth is used to get the location coordinates from a mobile phone that uses GPS for location services. An android app for searching the source to destination route. The control unit vibrates according to the route to inform the motorcyclist to take a left/right turn after a certain distance or to keep on heading straight or to take a U-Turn.
\end{abstract}

\section{General Terms}

Smartphones, Google Maps, Bluetooth, GPS.

\section{Keywords}

Navigation, Android, Route planning, Wearable

\section{INTRODUCTION}

In the present society utilizing an advanced mobile phone is ordinary. This project presents a prototype model and a system concept to provide smart shoes for bikers/cyclists. This system is intended to provide real-time Assistance system consist of microcontroller and a smartphone (GSM Module) and vibratory circuit. This project aims at the development of an smart shoes kit to help the bikers/cyclists to guide path. Utilizing your mobile phones while driving causes diversion, bringing about risky impacts like mishaps and passing. Bikers/Cyclists utilizing mobile phones are four times more prone to be associated with mishap cases. Utilizing vehicle and current innovation, for example, Bluetooth for accepting calls and dealing with information, utilizing route frameworks while driving may likewise be the reason for diversion.

\section{LITERATURE SURVEY}

In [6] discussion about navigation system which makes use of Google Maps API is done. Google Maps API provides a number of utilities for adding individual content to the Google map and various web map applications can be explored based on Google Maps API. Assisted global positioning systems (AGPS) is adopted by mobile operation companies as the highest accurate positioning way in mobile location based services. This paper proposes a solution of mobile navigation system that realizes such functions as Google map browse and query, bus lines search, the rapid local positioning on your mobile phone etc. The results prove that the solution of mobile navigation system based on Google Maps is feasible and valid, and it can be installed in PDA, mobile phones and other portable information terminals.

\author{
Dhananjay Gawari \\ PCCOE, Akurdi \\ Pune, Maharashtra, India
}

In [5] authors has three major contributions. First, a vehicular ad-hoc network (VANET)-based A* (VBA*) route planning algorithm is proposed to calculate the route with the shortest travelling time or the least oil consumption, depending on two real-time traffic information sources. The first one is the recorded traffic information of the road segment that the vehicle has passed through. This traffic information is further exchanged between vehicles when they enter the transmission range of IEEE $802.11 \mathrm{p}$ wireless link in the VANET. The second one is the traffic information provided by Google Maps. Then, a GPS navigation app is implemented on the Android platform to realize the VBA* route planning algorithm. Finally, simulations for six route planning algorithms are executed by the well-known VANET simulator, i.e., The ONE. In summary, VBA* achieves significant reductions on both the average travelling time and oil consumption of the planned route, as compared to traditional route planning algorithms.

\section{SYSTEM}

In our system we design a shoe that navigates the route from source to destination. Since the system is implemented in shoes we used a battery for power supply. Bluetooth is used to get the location coordinate from mobile phone by using GPS setting from mobile. Need an android app for searching the route destination to source route.

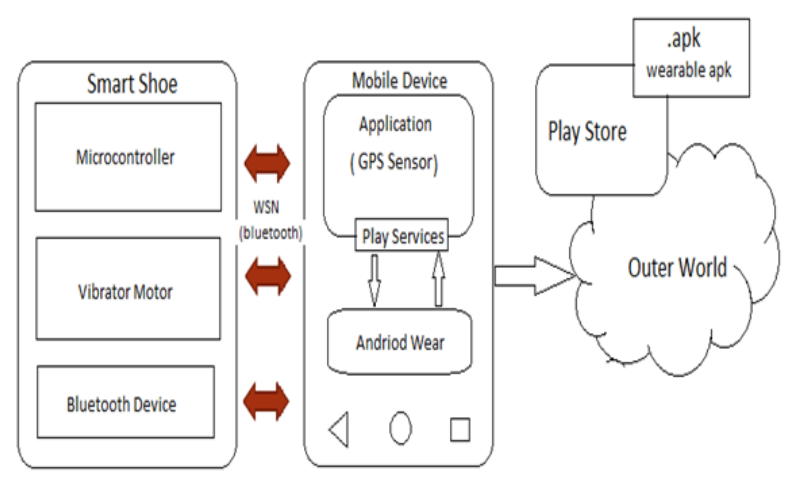

Fig 1: System Architecture

In this system novel technique to assist bikers/cyclists to track route in efficient way. The shoes sync up with a smartphone app that uses maps and vibrate to tell users when and where to turn to reach their destinations. When we need to drive on obscure street or we need to discover our goal for this reason we are utilizing GPS for course following however it is not helpful to utilize GPS amid the driving. So to overcome this issue we are accompanying new innovation which will fulfill our trip to following the course. Our venture would rotate 
around thinking of a navigational shoe model that could match with cell phone utilizing Bluetooth and help to give navigational data through vibration unit put all around shoe. Our undertaking would rotate around thinking of a keen shoe model that could combine with advanced cell utilizing Bluetooth and help to give navigational data through vibration unit set all around shoe. Basically, these shoes could give signs about when to proceed, where to go ahead and what kind of swing to take (left or ideal) to individual wearing these navigational shoes.

Our control unit gives vibration according to the route coordinates in shoes to indicate motorcyclist. So the motor cyclist moves in the direction of the vibration. Single Bikers/Cyclists like riders and bikers utilize bike.In such case it is exceptionally unpredictable assignment to see a mobile phone on an opportune premise. Henceforth require route without utilizing much phone. navigational shoe is a shoe which gives you the bearings in like manner. Vibrations are furnished with the assistance of which client come to know where to go ahead. GPS i.e. worldwide situating framework assumes a key part in this. To begin with client needs to set the goal on his telephone and tap the begin driving catch, naturally it will take client's present area as source i.e. begin point for drive and begins giving esteems. The scope and longitude of client is figured to know the present position after he/she begins moving. The estimations of scope and longitude bringing from GPS and gave to the advanced mobile phone. An advanced cell needs to associate with shoes for association. A microcontroller is settled in shoe which is in charge of further handling. Subsequent to getting esteems from PDA, microcontroller forms the information and as needs be client gets vibrations accordingly. Right cultivator will vibrate when right swing should be taken and left shoe will vibrate when left swing should be take.

NAVIGATIONAL VIBRATION SHOES FOR MOTORCYCLIST

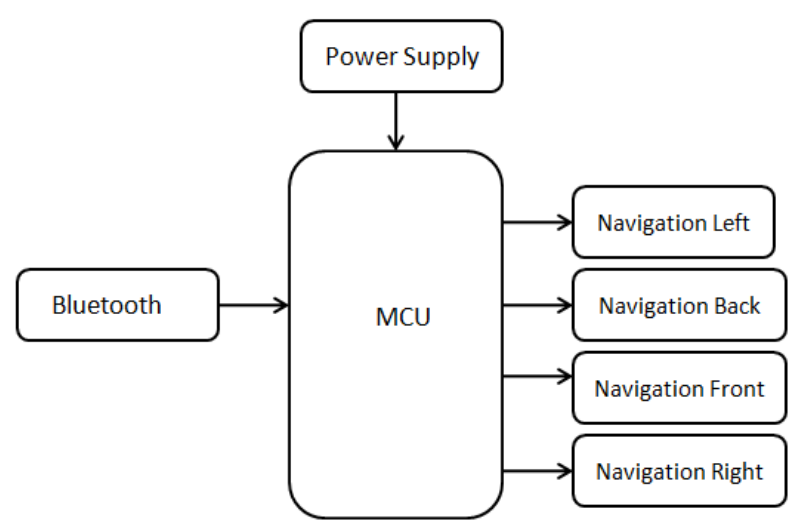

Fig 2: Smart Shoe Architecture

\section{ANDROID APP LAYOUT}

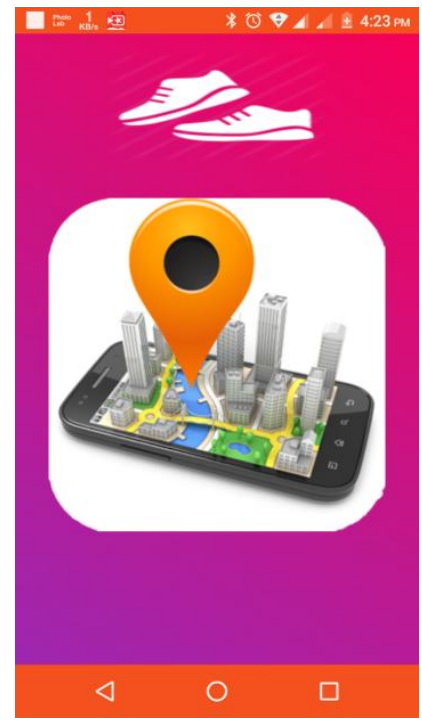

Fig 3: Splash Screen

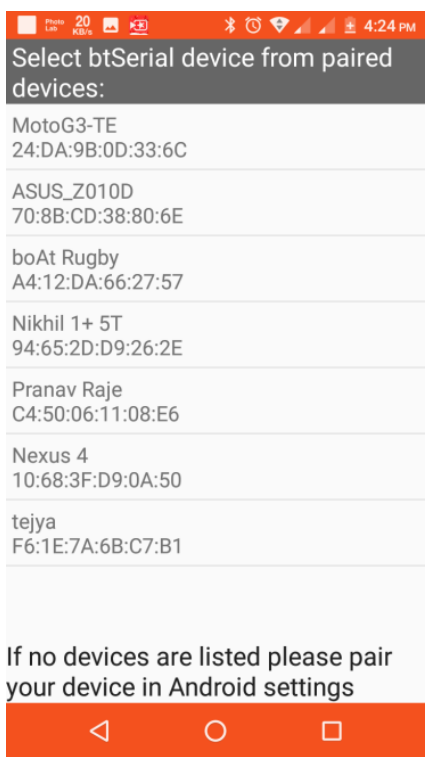

Fig 4: List of Available Bluetooth Devices
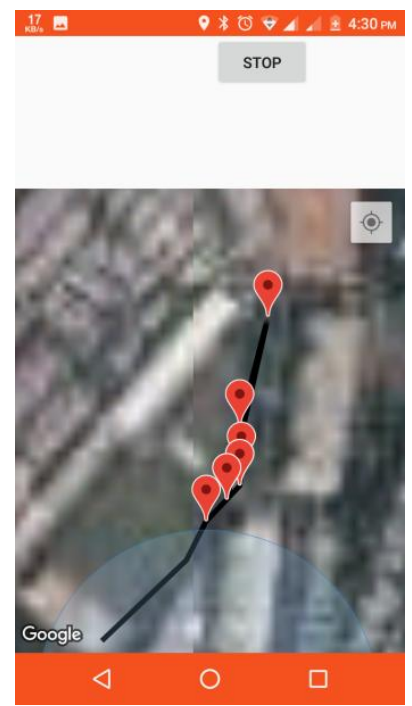

Fig 5: Map showing route selected by user 


\section{PROTOTYPING}

\subsection{PCB Design Process}

There are several basic steps involved in producing a printed circuit board (PCB). Most designs begin with a hand drawn schematic and design plan. With these, the circuit is prototyped and tested to verify that the design works correctly. Then, using software, an electronic version of the schematic is created. A netlist file is created from the electronic schematic and used in other software to create the physical layout of the PCB. Next, the components are placed and routed in the physical layout software and Gerber files are created. These Gerber files are used in a prototyping system to mill, drill, and cut the PCB substrate. The components are then placed and soldered to the substrate. Finally, the board is tested to verify that it works as expected. The major steps in the PCB design and fabrication process are as follows: 1 . design and test the prototype circuit - by hand; 2 . capture the circuit schematic - using or CAD Capture or similar software; 3 . perform the physical layout of the circuit- using or CAD Layout or similar software; 4. fabricate, populate and test the PCB - done by ECE shop personnel or similar personnel.

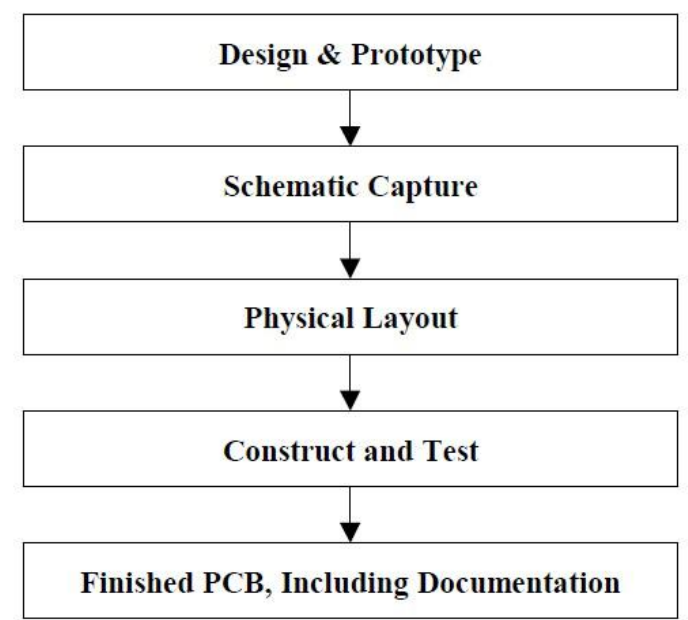

Fig 6: PCB design process

\section{HARDWARE}

\subsection{Arduino Uno}

Arduino/Genuine Uno is a microcontroller board based on the ATmega328P (datasheet). It has 14 digital input/output pins (of which 6 can be used as PWM outputs), 6 analog inputs, a $16 \mathrm{MHz}$ quartz crystal, a USB connection, a power jack, an ICSP header and a reset button. Connect it to a computer with a USB cable or power it with a AC-to-DC adapter or battery to get started.

Technical specification

- Microcontroller

- ATmega328

- Operating Voltage $5 \mathrm{~V}$

- Input Voltage (recommended) 7-12V

- Input Voltage (limit) 6-20V

- Digital I/O Pins 14 (of which 6 provide PWM output

- $\quad$ PWM Digital I/O Pins 6
- Analog Input Pins 6

- DC Current per I/O Pin $20 \mathrm{~mA}$

- $\quad$ DC Current for 3.3V Pin $50 \mathrm{~mA}$

- Flash Memory $32 \mathrm{~KB}$ (Atmega328P) of which 0.5 $\mathrm{KB}$ used by boot loader.

- $\quad$ SRAM 2 KB (ATmega328P)

- EEPROM $1 \mathrm{~KB}$ (ATmega328P)

- Clock Speed $16 \mathrm{MHz}$

- LED_BUILTIN 13

- Length $68.6 \mathrm{~mm}$

- Width $53.4 \mathrm{~mm}$

- Weight $25 \mathrm{~g}$

- Power:

The Arduino/ Genuine Uno board can be powered via the USB connection or with an external power supply. The power source is selected automatically. External (non-USB) power can come either from an AC-to-DC adapter (wall-wart) or battery. The adapter can be connected by plugging a $2.1 \mathrm{~mm}$ center-positive plug into the board's power jack. Leads from a battery can be inserted in the GND and Vin pin headers of the POWER connector. The board can operate on an external supply from 6 to 20 volts. If supplied with less than $7 \mathrm{~V}$, however, the $5 \mathrm{~V}$ pin may supply less than five volts and the board may become unstable. If using more than $12 \mathrm{~V}$, the voltage regulator may overheat and damage the board. The recommended range is 7 to 12 volts.

The power pins are as follows:

Vin. The input voltage to the Arduino/Genuino board when it's using an external power source (as opposed to 5 volts from the USB connection or other regulated power source). You can supply voltage through this pin, or, if supplying voltage via the power jack, access it through this pin.

$5 \mathrm{~V}$.This pin outputs a regulated $5 \mathrm{~V}$ from the regulator on the board. The board can be supplied with power either from the DC power jack $(7-12 \mathrm{~V})$, the USB connector $(5 \mathrm{~V})$, or the VIN pin of the board

(7-12V). Supplying voltage via the $5 \mathrm{~V}$ or $3.3 \mathrm{~V}$ pins bypasses the regulator, and can damage your board. We don't advise it.

3 V3. A 3.3 volt supply generated by the on-board regulator. Maximum current draw is $50 \mathrm{~mA}$.

GND. Ground pins.

IOREF. This pin on the Arduino/Genuino board provides the voltage reference with which the microcontroller operates. A properly configured shield can read the IOREF pin voltage and select the appropriate power source or enable voltage translators on the outputs to work with the $5 \mathrm{~V}$ or $3.3 \mathrm{~V}$. 


\subsection{Components}

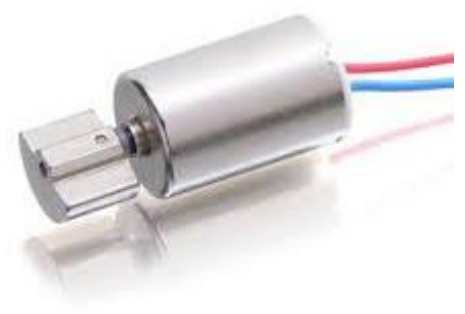

Fig 7: Micro Vibrator

A DC micro motor is any of a class of rotary electrical machines that converts direct current electrical energy into mechanical energy. The most common types rely on the forces produced by magnetic fields. Nearly all types of DC motors have some internal mechanism, either electromechanical or electronic, to periodically change the direction of current flow in part of the motor.

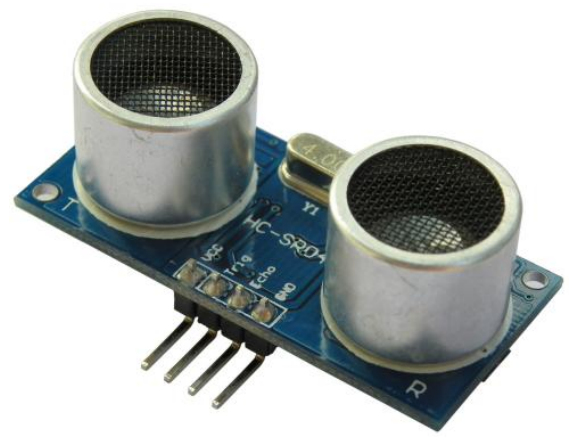

Fig 8: Ultrasonic Sensor

Ultrasonic Sensor : A Ultrasonic sensor is a device that can measure the distance to an object by using sound waves. It measures distance by sending out a sound wave at a specific frequency and listening for that sound wave to bounce back. By recording the elapsed time between the sound wave being generated and the sound wave bouncing back, it is possible to calculate the distance between the sonar sensor and the object.

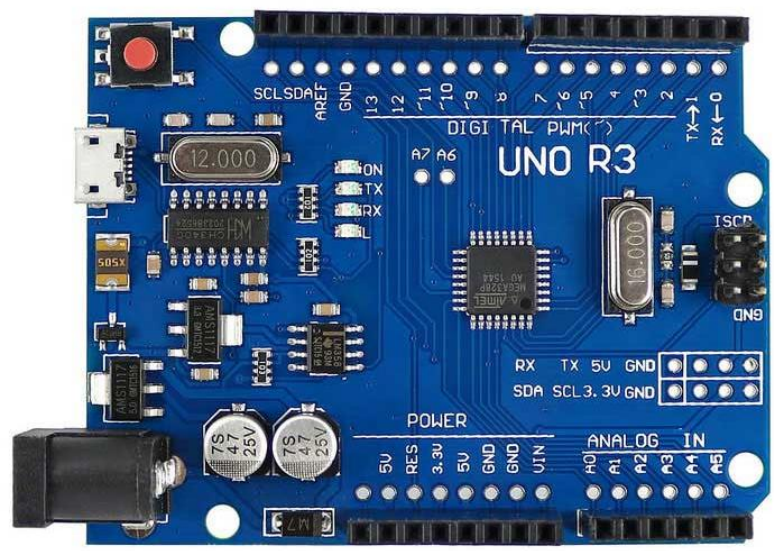

Fig 9: Arduino Uno

The Arduino UNO is a widely used open-source microcontroller board based on the ATmega328P microcontroller and developed by Arduino.cc. The board is equipped with sets of digital and analog input/output (I/O) pins that may be interfaced to various expansion boards (shields) and other circuits. The board features 14 Digital pins and 6 Analog pins. It is programmable with the_Arduino IDE via a type B USB cable. It can be powered by a USB cable or by an external 9 volt battery, though it accepts voltages between 7 and 20 volts.

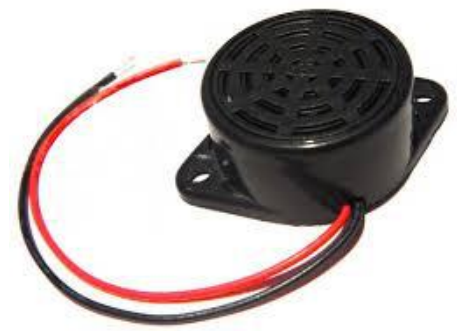

Fig 10: Buzzer

Buzzer : A buzzer or beeper is an audio signaling device, which may be mechanical, electro mechanical, or piezoelectric. Typical uses of buzzers and beepers include alarm devices, timers and confirmation of user input such as a mouse click or keystroke. Buzzer is an integrated structure of electronic transducers, DC power supply, widely used in computers, printers, copiers, alarms, electronic toys, automotive electronic equipment, telephones, timers and other electronic products for sound devices. Active buzzer $5 \mathrm{~V}$ Rated power can be directly connected to a continuous sound, this section dedicated sensor expansion module and the board in combination, can complete a simple circuit design, to "plug and play."

\section{WORKING}

In the present society using an advanced mobile phone is ordinary. This project presents a prototype model and a system concept to provide a smart shoe for driver. This system is intended to provide overall measures object detection, human detection, and real-time Assistance system consist of microcontroller, ultrasonic sensor and a smartphone (GSM Module) and vibratory circuit. This project aims at the development of an smart shoes kit to help the driver to guide path. Utilizing your mobile phones while driving causes diversion, bringing about risky impacts like mishaps and accidents. Drivers utilizing mobile phones are four times more prone to be associated with mishap cases. Utilizing vehicle and current innovation, for example, Bluetooth for accepting calls and dealing with information, utilizing route frameworks while driving may likewise be the reason for diversion.

\section{ADVANTAGES}

1) The comparably higher accuracy and better comprehensibility, simplicity and low weight of a actuator System.

2) does not require users to hold or carry their smartphones in specific ways in order to be able to navigate properly.

3) Navigation Assistance while travelling.

4)Distraction-free travel.

5) Automatic rerouting and alerts.

6)Various User-controlled Vibration pattern.

7) User friendly system

\section{CONCLUSION}

Our System introduces a general framework for checking courses for navigational purposes, which may be obscure or unknown to the user. The framework comprises of microcontroller, vibrators/actuators , Bluetooth module and an 
Android application, which leverages the built-in GPS functionality that is commonly found in an average Android smartphone. The Smart Shoe System is a kind of wearable device. Shoe is one of the few things that a person does not go out without, thus using the smart navigational shoes system is a very natural experience, unlike other solutions and can be used with ease, without distracting the users. The system can be extended to help visually challenged people for navigational purposes, using voice commands for input. Also the system can be extended to integrate the application with Google assistant, as well as provide various metrics like calories burned, count of steps, etc.

\section{REFERENCES}

[1] Ying-Xun Lai, Yi-Wei Ma1, Yueh-Min Huang1, JiannLiang Chen, and Subhas Chandra Mukhopadhyay." Ubiquitous Motion Sensing Service Using Wearable Shoe Module and Mobile Device", 1National Cheng Kung University, Tainan, Taiwan 2016.

[2] Miss. Smita S. Auti*, Prof. Nagnath B. Hulle," Advanced shoes with embedded position tracking and path guidance to keep track of Alzheimer's patients", Electronics \& Telecommunication Dept., GHRCOEM, Chas, Ahmednagar, India, January 2015.

[3] Diksha M. Wasekar ,Purva D. Thakare , Prof. R. P. Bijwe ," Lechal Footwear Technology", Associate Professor CSE, H.V.P.M's COET Amravati, Maharashtra, India, April 2016.

[4] Battin, Pradnya, and S. D. Markande. "Location based reminder Android application using Google Maps API."
Automatic Control and Dynamic Optimization Techniques (ICACDOT), International Conference on. IEEE, 2016

[5] Chang, Chau, et al. "Design and implementation of the travelling time-and energy- efficient android gps navigation app with the vanet-based $a^{*}$ route planning algorithm." Biometrics and Security Technologies (ISBAST), 2013 International Symposium on. IEEE, 2013.

[6] Li, He, and Lai Zhijian. "The study and implementation of mobile GPS navigation system based on Google Maps." Computer and Information Application (ICCIA), 2010 International Conference on. IEEE, 2010.

[7] Rahman, Md Marufi, et al. "Real time Google map and Arduino based vehicle tracking system." Electrical, Computer \& Telecommunication Engineering (ICECTE), International Conference on. IEEE, 2016.

[8] Erol, Mehmet Hamza, and Faruk Bulut. "Real-time application of travelling salesman problem using Google Maps API." Electric Electronics, Computer Science, Biomedical Engineering's' Meeting (EBBT), 2017. IEEE, 2017.

[9] Rosen S. lvanov "Algorithm for GPS Navigation, Adapted for Visually Impaired People".

[10] Li Yang, "Smart Shoe": an autonomous inkjet-printed RFID system scavenging walking energy" 2011 